\title{
CANDIDA CONUNDRUMS: USE OF NYSTATIN IN AZOLE RESISTANT CANDIDA ISOLATES
}

\author{
27-30 June, Dublin Ireland, IUSTI World and European Congress
}

Dr Harriet Le Voir, Dr Jon Lambourne, Dr Liat Sarner, Royal London Hospital, Barts Health NHS Trust

\section{Background:}

Vulvovaginal candidiasis is a common cause of vaginal discharge and genital itching affecting the majority of women at least once before the age of 55 . Candida albicans is responsible for approximately $90 \%$ of infections ${ }^{1}$ and Candida glabrata the most common non-albicans type isolated ${ }^{2}$. The incidence of $C$. glabrata and azole resistant $C$. albicans is increasing ${ }^{3,4}$.

$5 \%$ of those affected with Candida species suffer with recurrent episodes (more than 4 episodes per year) 5,6 . Following suppressive treatment there can continue to be return of symptoms which may be due to relapse from persistent vaginal reservoir of Candida rather than infection with a new strain ${ }^{7}$.

\section{Methods:}

We evaluated the cases of 13 women with complicated, recurrent Candida who attended a specialised female problem clinic in a London Genito-urinary medicine clinic. Clinical data was extracted from electronic patient record systems. This included clinical and treatment history, mycological investigations and resultant optimised treatment regime.
Results:

13 women of multi-ethnicity were identified with an age range 23 to 51 years. 10 women had onset of their symptoms more than 1 year ago, with 7 out of 13 women having been treated unsuccessfully with 3 or more different candidiasis treatments. Reduced Candida susceptibility to azole treatments could be attributed to treatment failure in 10 of the 13 cases. C. glabrata was cultured in 5 women with azole resistance in 3 cases; 5 cases were identified as $C$. albicans with azole resistance; and 2 cases cultured other Candida species (C. krusei and $C$. nivariensis). 3 women were colonised with two species simultaneously.

Following sensitivity guided treatment regimens, 10 patients responded symptomatically and/or with negative culture results after treatment containing a nystatin (+/- flucytosine) regimen. 6 of those patients were also treated with an azole as part of the treatment regimen ( 3 as part of the initial regimen with nystatin; 3 as additional treatment with an azole due to return of symptoms after cessation of nystatin). The remaining 3 patients were treated with azoles (2 with clotrimazole following poor response to nystatin and 1 with itraconazole due to patient preference after partial response to nystatin).
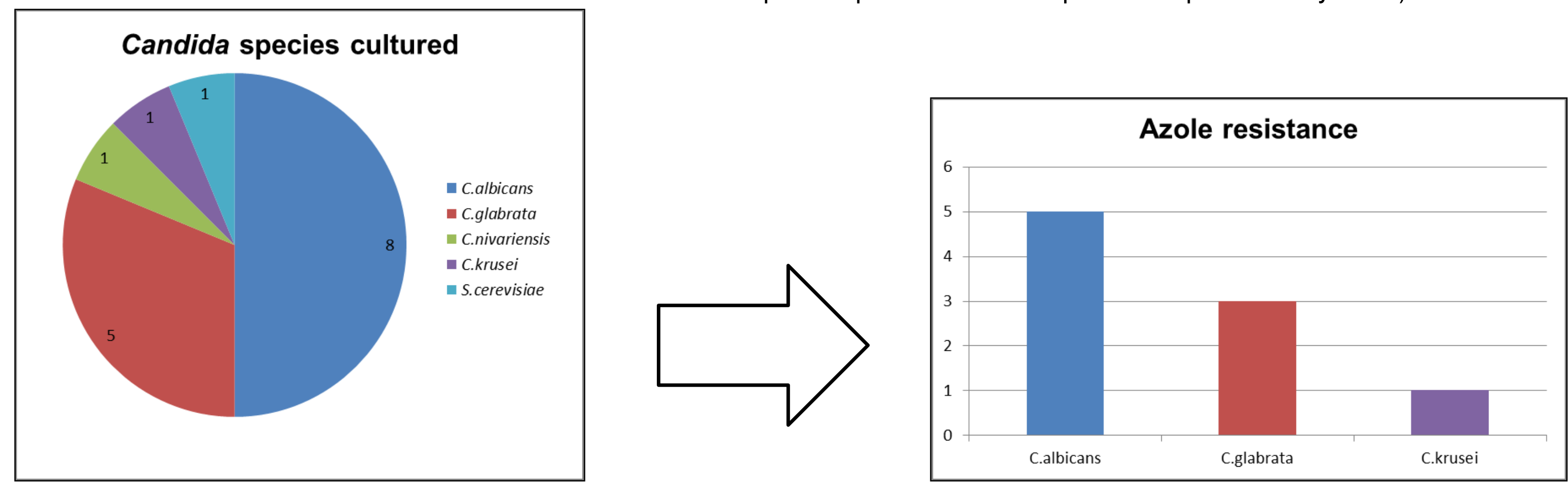

\begin{tabular}{|c|c|c|c|c|c|c|c|c|c|}
\hline & $\begin{array}{l}\text { Years since } \\
\text { symptom } \\
\text { onset }\end{array}$ & $\begin{array}{l}\text { Previous } \\
\text { regimens }\end{array}$ & Species & $\bar{S}$ & I & $\mathbf{R}$ & \multicolumn{2}{|l|}{ Optimised treatment following sensitivities } & $\begin{array}{r}\text { Outcome: } \\
\text { symptoms and culture }\end{array}$ \\
\hline 1 & 3 & 7 & C. albicans & $\mathrm{F}$ & & $\mathrm{F}$ & \multicolumn{2}{|l|}{$\mathrm{N} / \mathrm{FX}$ induction and $3 /$ week maintenance $6 / 12$} & $\begin{array}{l}\text { Symptoms controlled. Culture and slide } \\
\text { positive with incorrect use. }\end{array}$ \\
\hline 2 & 11 & 4 & C. glabrata & $\mathrm{MN}$ & IC & $\mathrm{F}$ & \multicolumn{2}{|l|}{$\begin{array}{l}P \text { od } 2 / 12 \& N \text { induction } \rightarrow N 3 / \text { week maintenance } \\
6 / 12\end{array}$} & $\begin{array}{r}\begin{array}{r}\text { Symptoms controlled, slide and culture } \\
\text { negative }\end{array} \\
\end{array}$ \\
\hline 3 & $2+$ & 1 & C. glabrata & IMN & & $\mathrm{F}$ & \multicolumn{2}{|l|}{$\begin{array}{l}\text { N/FX 2x induction } \rightarrow \text { symptoms returned } \\
\mathrm{C} \text { weekly pessary } 6 / 12\end{array}$} & $\begin{array}{l}\text { Culture negative but symptoms returned } \\
\text { and improved with azole. }\end{array}$ \\
\hline 4 & 3 & 2 & C. glabrata & $\mathrm{FI}$ & & & \multicolumn{2}{|l|}{ I od 14d $\rightarrow \mathrm{N} / \mathrm{FX}$ induction } & Symptoms controlled, culture negative \\
\hline 5 & 7 & 3 & C. albicans & FNC & IM & $\mathrm{F}$ & \multicolumn{2}{|l|}{$\begin{array}{l}\mathrm{N} / \mathrm{FX} \text { induction \& I od } 7 \mathrm{~d} \\
3 / 12 \text { later: } \mathrm{C} \text { pessary every } 72 \mathrm{~h} \rightarrow 3 / \text { week }\end{array}$} & $\begin{array}{r}\text { Symptom free for } 3 \text { months and then } \\
\text { culture positive. }\end{array}$ \\
\hline 6 & 1 & 3 & 1) C. glabrata & FIM & c & IF & \multicolumn{2}{|l|}{$\begin{array}{l}\mathrm{N} / \mathrm{FX} \text { induction } \rightarrow \text { symptoms returned. } \\
\text { F 150mg fortnightly }\end{array}$} & $\begin{array}{r}\text { Culture negative but symptoms returned } \\
\text { and improved with azole. }\end{array}$ \\
\hline 7 & 3 & 2 & C. albicans & $\mathrm{Cl}$ & M & $\mathrm{F}$ & \multicolumn{2}{|l|}{$\begin{array}{l}\text { I od stopped as deranged LFT. } \\
\mathrm{N} \text { induction \& weekly maintenance } 6 / 12\end{array}$} & Symptoms controlled, culture negative \\
\hline 8 & 3 & 2 & C. albicans & 1 & & $\mathrm{~F}$ & \multicolumn{2}{|l|}{$\mathrm{N}$ induction } & $\begin{array}{r}\text { Symptoms improved, no culture follow up } \\
\text { yet as on going. }\end{array}$ \\
\hline 9 & 17 & 3 & 1)S. cerevisiae & FIMNC & CFI & & \multicolumn{2}{|l|}{$\begin{array}{l}\mathrm{N} \text { induction slow relief. } \\
\mathrm{C} \text { weekly pessary } 6 / 12\end{array}$} & $\begin{array}{r}\text { Symptoms improved with azole, no culture } \\
\text { follow up yet as on going. }\end{array}$ \\
\hline 10 & 1 & 2 & C. albicans & INC & FM & & \multicolumn{2}{|l|}{$\mathrm{N}$ induction } & $\begin{array}{r}\text { Symptoms improved, no culture follow up } \\
\text { yet. }\end{array}$ \\
\hline 11 & $<1$ & 3 & $\begin{array}{l}\text { C. albicans } \\
\text { C. glabrata }\end{array}$ & $\mathrm{FI}$ & & & \multicolumn{2}{|l|}{$\begin{array}{l}N \text { induction and } 2 / \text { week maintenance partial relief. } \\
\text { I bd } 14 d \rightarrow \text { od } 2 / 12\end{array}$} & $\begin{array}{r}\text { Symptoms improved with azole, no culture } \\
\text { follow up yet as on going. }\end{array}$ \\
\hline 12 & 2 & 2 & C. nivariensis & $\mathrm{FI}$ & & & \multicolumn{2}{|l|}{$N$ induction no relief $\rightarrow C$ pessary $7 d$} & $\begin{array}{r}\text { Symptoms improved with azole, no culture } \\
\text { follow up as on going. }\end{array}$ \\
\hline 13 & $2+$ & 4 & C. krusei & NC & IM & & \multicolumn{2}{|l|}{$\mathrm{N} / \mathrm{FX}$ induction $\rightarrow$ 2/week maintenance $2 / 12$} & $\begin{array}{l}\text { Culture negative, symptoms persist, } \\
\text { referred immunology }\end{array}$ \\
\hline \multirow{2}{*}{\multicolumn{9}{|c|}{$\begin{array}{l}\text { Table 1: } \\
\mathrm{F}=\text { fluconazole, } \mathrm{I}=\text { itraconazole, } \mathrm{M}=\text { miconazole, } \mathrm{N}=\text { nystatin, } \mathrm{C}=\text { clotrimazole, } \mathrm{FX}=\text { flucytosine, } \mathrm{P}=\text { posoconazole } \\
\text { Nystatin/flucytosine induction regimen: } \mathrm{N} 100000 \text { units PV, FX } 1 \mathrm{~g} \mathrm{PV} \text { od } 14 \text { days } \\
\text { Nystatin maintenance: } \mathrm{N} 100000 \text { units } \mathrm{PV} 2-3 / \text { week }\end{array}$}} & $\begin{array}{l}\text { Response to nystatin regimen } \\
\text { Response and relapse }\end{array}$ \\
\hline & & & & & & & & & Poor response to nystatin \\
\hline
\end{tabular}

\section{Conclusion:}

Women with repeated episodes of vulvovaginal candidiasis where standard treatment is ineffective should have samples sent for mycological analysis at an early stage to prevent prolonged ineffective treatment and reduce the risk of further resistance to azoles. Minimum inhibitory concentrations (MIC) with sensitivity testing can guide effective treatments, however higher $\mathrm{pH}$ environments within the vagina may cause reduction in efficacy of treatments in-vivo, especially if isolates are at a threshold of resistance ${ }^{8}$. Based on serum drug level testing, the dose of azole was adjusted in case 2 to increase efficacy of treatment in view of MIC from sensitivity testing.

Patient preference and presence of symptoms is also is important when considering an effective regimen as intra-vaginal therapy is not tolerated by all women. Follow up of patients with culture should be utilised to identify treatment efficacy or failure.

For women with Candida species exhibiting azole resistance, topical nystatin therapy as an induction and maintenance treatment can be an effective regime which should be considered alongside optimisation of other contributory factors such as diabetes, hyperoestrogenic states, immunosuppression ${ }^{9}$. Combination regimens with nystatin and appropriate azoles utilising sensitivity results is also effective. 